\section{Diversity, Equity, and Representativeness: Coming to Terms With the Henrietta Lacks Act}

\author{
Daniel J. Boffa, MD; Keith B. Churchwell, MD; and Richard C. Maduka, MD
}

$\mathbf{M}$ any of us have read about the legacy of Henrietta Lacks, ${ }^{1}$ the African American woman whose immortalized cancer cells, harvested from her cervical tumor, went on to tremendously impact cancer research and medical science as a whole. Although the benefit of her contribution is widespread and difficult to measure, it is tainted by a lack of informed consent for tissue receipt, the segregation of the hospital in which she was receiving care, and the racial discrimination (still ongoing today) jeopardizing the management and overall outcomes of minority patients.

The recently passed Henrietta Lacks Enhancing Cancer Research Act will direct the Government Accountability Office to study and publish a report regarding barriers to participation in federally funded cancer clinical trials by populations that have been traditionally underrepresented in previous studies. ${ }^{2}$ If successful, the Act will help to broaden the access that "racial and ethnic minorities, older, rural and lower income individuals" have to cancer research trials. The following is an attempt to define, in simple terms, the concepts which made the Act necessary, and to estimate the Act's potential to impact underrepresented populations.

The Act addresses 3 connected concepts in healthcare policy: diversity, equity, and representativeness. Establishing a consensus definition of these terms is critical to understanding the potential impact of the Act. In simplest terms, patient diversity refers to the degree to which patients receiving care differ from one another. Diversity is a complicated concept in medical research. On the one hand, a lack of uniformity in experimental conditions allows for alternate explanations of study results (eg, effectiveness of tyrosine kinase inhibitors as cancer treatments may altered by some medications for gastroesophageal reflux). ${ }^{3}$ On the other hand, diversity of study subjects is critical to ensure that research findings are valid for a broad population of patients under a variety of different "real-world" conditions. One thing that is clear-not all groups are currently considered equally in cancer research.

Equity relates to results brought about by a deliberate distribution of resources. In healthcare, equity means that every person has the same opportunity to achieve their best possible health outcome. Equity does not require everyone to achieve the same outcome. Because each patient represents a unique set of conditions and circumstances capable of influencing outcomes, as well as potentially unique goals of care, each patient has their own best possible outcome. Equity in healthcare does not mean everyone is given the same resources (ie, physician time, medications, etc), because individuals may require different levels of support to achieve the same level of opportunity for health. Consider a grove of fruit trees in which the lowest fruit is 8 feet off the ground and a truck containing different-sized stepladders. In order to create an equitable opportunity to access the fruit, individuals would be given the appropriate-sized ladder for their height and reach. Obviously, the "fruit" in this analogy relates to optimal health, while the ladders relate to healthcare resources. A lack of equity, or situation in which a group is consistently achieving a different level of opportunity, is a disparity (eg, ladders not being given to

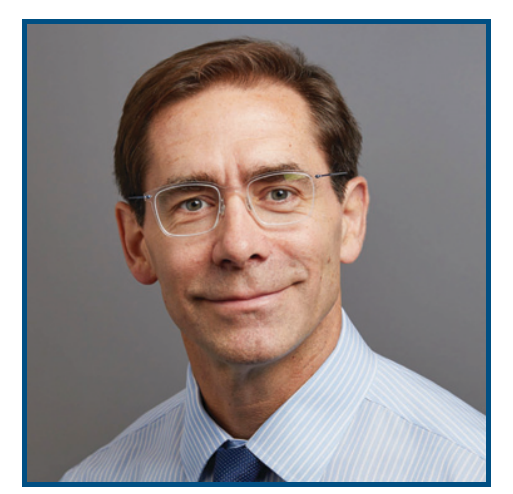

DANIEL J. BOFFA, MD

Daniel J. Boffa, MD, is a Professor and Chief of Thoracic Surgery at Yale University, and is the Clinical Director of the Thoracic Oncology Program at Smilow Cancer Hospital.

He grew up in central Ohio, trained in New York City and Cleveland, and has been at Yale since 2007. He completed his MBA from the Heller School of Social Policy and Management in 2021.

His clinical practice focuses on the surgical management of thoracic malignancies. His research interests include optimizing the safety and effectiveness of cancer treatments, the potential of hospital networks to impact care delivery, and the impact of health policy initiatives on patient care.

doi: 10.6004/jnccn.2021.7071

The ideas and viewpoints expressed in this commentary are those of the author and do not necessarily represent any policy, position, or program of NCCN. 


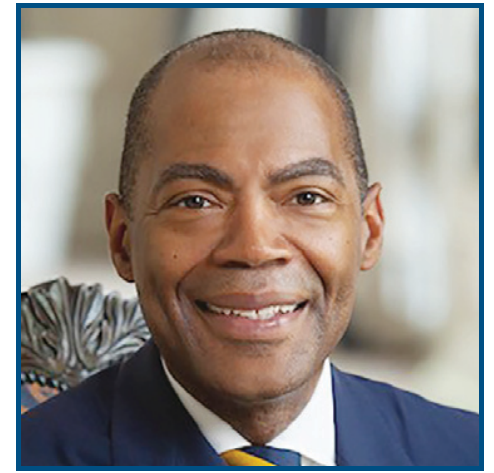

\section{KEITH B. CHURCHWELL, MD}

Keith B. Churchwell, MD, is the President of Yale New Haven Hospital and Associate Clinical Professor of Medicine at Yale School of Medicine.

He obtained his undergraduate degree from Harvard University and medical degree from Washington University School of Medicine. He spent his formative training years at Emory University Medical School and Affiliated Hospitals including a fellowship in cardiovascular medicine and nuclear cardiology. Before his time at Yale New Haven, he spent 16 years at the Vanderbilt School of Medicine and Medical Center as a clinical cardiologist and an associate professor of medicine and radiology. He also worked in a number of administrative positions over the years culminating in leading the Heart and Vascular Institute on campus as its Executive Director and Chief Medical Officer for 6 years. people over a certain age). Importantly, improving equity should not mean compromising the opportunity of others, because they often need different types of support. In the grove analogy, this could equate to holding someone's hand as they climb the ladder. Finally, equity is not the same as fairness. In fact, sometimes a fair distribution can undermine equity. Randomly assigning ladders would be fair, but would not result in equity.

Representativeness refers to the degree to which a sample resembles the population from which it originates. ${ }^{4}$ To ensure that sampled information is applicable to the larger group, the sample must include the key attributes of the population. In the fruit grove analogy, a representative sample must include people of varying heights to ensure the appropriate range in ladder sizes are available. These terms frame important questions over whether better access to translational research will translate into better cancer care.

Can the Act improve diversity? If the Act works as intended, it will expand access of federally funded clinical cancer trials to a broader diversity of participants. It is unclear if the enhancement of diversity in research will improve diversity more broadly within cancer care.

Will the Act improve healthcare equity? The Act is designed to increase access to clinical trials for underrepresented populations (ie, research equity). Clinical trials may allow patients the opportunity to receive a superior treatment before it is widely available. In this regard, equity in trial access could enhance healthcare equity, at least to the extent that trials include a true advance in care. Historically, approximately $5 \%$ of clinical trials have resulted in the approval of a new cancer treatment (more recently closer to $10 \%) .{ }^{5}$ Although this may be a small fraction of trials, one must keep in mind that trials tend to involve more patients as they progress through the stages of approval, improving the odds that trial participation is beneficial. As such, research equity may, to a limited degree, directly enhance healthcare equity by enabling underrepresented populations better access to superior treatments before the treatments become the standard of care.

Will the Act improve representativeness? Because no two patients with cancer are identical, cancer research is designed to capture the average effect of an intervention across a population. To estimate the average effect, clinical trials must study patient subsets that are sufficiently similar to individuals needing the intervention being studied (ie, representative). How "similar" is "sufficient"? At a minimum, one hopes to include attributes of a population that would change the average effect. Ideally, trials should aspire to generate data that are relevant to the vast majority of people needing the studied intervention, which requires far broader representation. By increasing access to clinical trials, the Act should increase the representativeness of cancer research for certain important attributes of the underrepresented cohort. For example, minoritized race status is more common in the underrepresented populations, and important cancer findings have been linked to genetic ancestry. ${ }^{6}$ As an example, the EGFR mutation in lung cancer is substantially more common in never-smoking women of Asian descent. ${ }^{7,8}$ Inclusion of this demographic subset was imperative to make this critical observation. The age of onset for screen-detectable cancers varies across populations whose ancestry is tied to different parts of the world. ${ }^{8}$ Failure to adequately represent genetic ancestral diversity and social determinants in cancer screening trials could result in the age of first screening examinations being set inappropriately high, causing cancers to be missed. In fact, addressing this phenomenon in cigarette smokers was one of the motivations for the recent expansion in eligibility criteria for lung cancer screening. ${ }^{9,10}$ Furthermore, the attempt to diversify research 
populations could improve the representation of several critical social determinates, such as environmental and nutritional factors, which are major contributors to the health of the underrepresented populations. ${ }^{11}$ Therefore, the Act should increase the representativeness of several important attributes of underrepresented populations.

On the other hand, it is important to remember that disparities in patient outcomes likely stem from multiple facets of our healthcare system. The Act may obscure some of the more important issues impacting the health of underrepresented populations by changing/augmenting the sampled population before they are studied. Specifically, by mitigating barriers to trial participation, the Act will enable underrepresented populations to be maximally compliant with trial care (a good thing). The attribute that is the most prognostic/problematic for many patients in these cohorts may be the lack of resources, access, or information required to fully participate in recommended care or earned mistrust of healthcare systems and science. Ironically, for some minoritized factions, the same barriers the Act is attempting to remove from cancer research participation may also prevent similar patients from benefiting from research findings. If only the trial patients are provided resources, the same barriers could prevent similar patients from partaking in the intervention the research identified as being beneficial. In the fruit grove analogy, this would be akin to augmenting the sampled population by giving individuals in the sample set with the shortest reach a rake to pull fruit from higher branches. This intervention could obscure the need of this subgroup for taller stepladders within the larger population. Therefore, care must also be studied as it occurs outside of clinical trials, to fully understand the performance of interventions under real-world conditions.

Representativeness and equity are destinations that are far easier to know when you are not there than when you have arrived. The Act is a good step toward addressing a great need, but few journeys are completed in a single step. The same findings pertaining to the barriers and mitigators to trial participation could facilitate care participation in general. Hopefully stakeholders will leverage this information to more broadly address the diverse needs within our diverse populations so that all may achieve their optimal cancer outcomes. Ultimately, the Act is not the end of a problem but the beginning of a solution.

Disclosures: The authors have disclosed that they have no financial interests, arrangements, or affiliations with the manufacturers of any products discussed in this article or their competitors.

Correspondence: Daniel J. Boffa, MD, 330 Cedar Street, BB205, PO Box 208062, New Haven, CT 06520 8062. Email: daniel.boffa@yale.edu

\section{References}

1. Skloot R. The Immortal Life of Henrietta Lacks. Crown Publishing Group; New York: 2010.

2. Henrietta Lacks Enhancing Cancer Research Act of 2019, HR 1966, 116th Congress (2019-2020). Pub L No. 116-291. Accessed January 25, 2021. Available at: https://www.congress.gov/bill/116thcongress/ house-bill/1966

3. van Leeuwen RWF, Jansman FGA, Hunfeld NG, et al. Tyrosine kinase inhibitors and proton pump inhibitors: an evaluation of treatment options. Clin Pharmacokinet 2017;56:683-688.

4. Kahneman D, Tversky A. Subjective probability: a judgment of representativeness. Cognit Psychol 1972 ; 3:430-454.

5. Wong $\mathrm{CH}$, Siah KW, Lo AW. Estimation of clinical trial success rates and related parameters. Biostatistics 2019;20:273-286.

6. Perez NP, Baez YA, Stapleton SM, et al. Racially conscious cancer screening guidelines: a path toward culturally competent science [published online May 19, 2020]. Ann Surg, doi: 10.1097/SLA. 00000000000039837

7. Wu YL, Cheng Y, Zhou X, et al. Dacomitinib versus gefitinib as first-line treatment for patients with EGFRmutation-positive non-small-cell lung cancer (ARCHER 1050): a randomised, open-label, phase 3 trial. Lancet Oncol 2017;18:1454-1466.

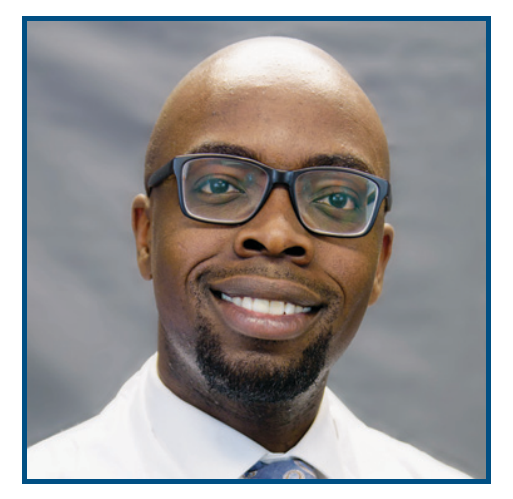

RICHARD C. MADUKA, MD

Richard C. Maduka, MD, is a resident of the Yale New Haven Hospital General Surgery Program.

A native of Prince Georges County Maryland, he obtained his undergraduate degree in Chemistry as a Meyerhoff Scholar at the University of Maryland, Baltimore County. He then went on to receive his medical degree at the Perelman School of Medicine at the University of Pennsylvania.

Currently as a surgical resident, he works with Dr. Daniel J. Boffa to describe and alleviate disparities in the oncologic care of patients. 
8. Midha A, Dearden S, McCormack R. EGFR mutation incidence in nonsmall-cell lung cancer of adenocarcinoma histology: a systematic review and global map by ethnicity (mutMaplI). Am J Cancer Res 2015;5: 2892-2911.

9. Krist AH, Davidson KW, Mangione CM, et al. Screening for lung cancer: US Preventive Services Task Force recommendation statement. JAMA 2021; 325:962-970.
10. Aldrich MC, Mercaldo SF, Sandler KL, et al. Evaluation of USPSTF lung cancer screening guidelines among African American adult smokers. JAMA Oncol 2019;5:1318-1324.

11. Office of Disease Prevention and Health Promotion. Social Determinants of Health. Accessed February 24, 2021, 2021. Available at: https://www. healthypeople.gov/2020/topics-objectives/topic/social-determinants-ofhealth
NCCN Global Academy for Excellence \& Leadership in Oncology ${ }^{\mathrm{Tm}}$

School of Pharmaceutical \& Biotech Business

\section{Learn about NCCN's Global Resources and Meet NCCN Guidelines ${ }^{\circledR}$ Panel Members and Collaborative Experts! \\ Register Now \\ 23 September 2021 | Virtual Program}

NCCN.org/globalacademy 